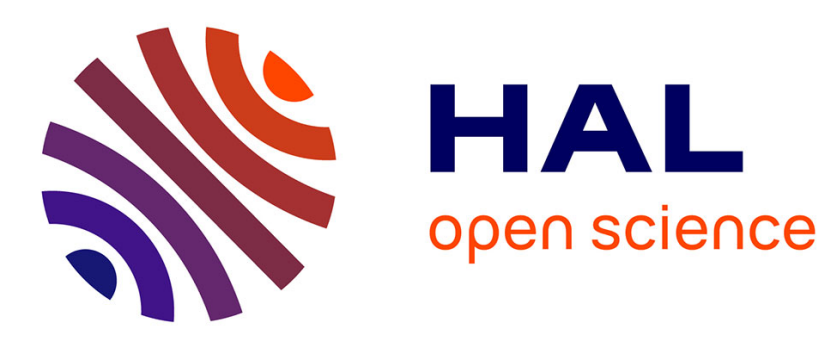

\title{
Audio watermarking: a way to stationnarize audio signals
}

Sonia Djaziri-Larbi, Mériem Jaidane

\section{To cite this version:}

Sonia Djaziri-Larbi, Mériem Jaidane. Audio watermarking: a way to stationnarize audio signals. IEEE Transactions on Signal Processing, 2005, 53 (2), pp.816-823. 10.1109/TSP.2004.839899 hal02520660

\section{HAL Id: hal-02520660 https://hal.science/hal-02520660}

Submitted on 26 Mar 2020

HAL is a multi-disciplinary open access archive for the deposit and dissemination of scientific research documents, whether they are published or not. The documents may come from teaching and research institutions in France or abroad, or from public or private research centers.
L'archive ouverte pluridisciplinaire HAL, est destinée au dépôt et à la diffusion de documents scientifiques de niveau recherche, publiés ou non, émanant des établissements d'enseignement et de recherche français ou étrangers, des laboratoires publics ou privés. 


\title{
Audio Watermarking: A Way to Stationnarize Audio Signals
}

\author{
Sonia Larbi and Mériem Jaïdane
}

\begin{abstract}
Audio watermarking is usually used as a multimedia copyright protection tool or as a system that embed metadata in audio signals. In this paper, watermarking is viewed as a preprocessing step for further audio processing systems: the watermark signal conveys no information, it is rather used to modify the statistical characteristics of an audio signal, in particular its non stationarity. The embedded watermark is then added in order to stationnarize the host signal. Indeed, the embedded watermark is piecewise stationary, thus it modifies the stationarity of the original audio signal. In some audio processing fields, this fact can be used to improve performances that are very sensitive to time-variant signal statistics.

This paper presents an analysis of the perceptual watermarking impact on the stationarity of audio signals. The study is based on stationarity indices, which represent a measure of variations in spectral characteristics of signals, using time frequency representations. Simulation results with two kinds of signals, artificial signals and audio signals (speech and music) are presented. Stationarity indices comparison between watermarked and original audio signals shows a significant stationarity enhancement of the watermarked signal, especially for transient attacks.
\end{abstract}

Index Terms-Perceptual audio watermarking, stationarity indices, time frequency representations.

\section{INTRODUCTION}

A UDIO watermarking is usually used as a multimedia copyright protection tool or as a system that embed metadata in audio signals. The current challenge is that these data hiding techniques are robust to removal attacks and standard signal processing manipulations. Obviously, to be effective, the watermark must be imperceptible within its host. This is done by exploiting the masking property of the human auditory system (see for example [1]).

In this paper, audio watermarking is analyzed from an unusual point of view, where the information content of the watermark is rather irrelevant (although it could be), whereas its statistical characteristics are the most important determinant for the purpose of the proposed preprocessing. The watermark signal is viewed as a way to modify the statistical characteristics of audio signals, in particular its non stationarity.

Indeed, in various audio processing fields, performances are very sensitive to time variant signal statistics. For example, in acoustic echo cancelation, many adaptive algorithms are developed in order to be robust to such non stationarities (see for example [2]). They do a trade-off between short time convergence and small residual echo.

S. Larbi and M. Jaïdane are with the Communications and Information Technologies Department at Ecole Nationale d'Ingénieurs de Tunis, Tunisia. (e-mail: Sonia.Larbi@enit.rnu.tn; nej.jaidane@planet.tn

This work was presented at the IEEE ICASSP Conference, Hong Kong, April 2003.
In noise reduction, the sudden changes in signal characteristics cause frequent misadjustment of the denoising algorithm. By another way, audio coding operates on a per-frame basis to overcome signal non stationarity, where the frame length is a trade-off between coding efficiency and pre-echo avoidance. Abrupt temporal variations of the waveforms limit low bit rate coding [3].

In some applications, it is possible to drive the processing system by the watermarked audio signal instead of the original. Indeed, in [4] the authors propose a solution to the problem of the correlation between the loudspeaker signals in a stereophonic acoustic cancelation environment, which results in an ill-conditioned system. They add a spectrally shaped white noise to the loudspeaker input signals. The principle of this solution is very similar to that of perceptual spread spectrum watermarking [1].

In this paper, we propose an analysis of the watermarking influence on the stationarity of audio signals [5]. The present study is motivated by a previous work on robustness enhancement of a monophonic adaptive echo canceler driven by the watermarked speech instead of the original [6].

The considered time domain watermarking scheme is shown on Fig. 1. The embedded watermark signal $t_{n}$ is synthesized by spectral shaping of the stationary random sequence $v_{n}$, which represents the signature in the watermarking context. This is done through an allpole filter $H(z)$, using perceptual properties of the human ear. The watermark $t_{n}$ is then embedded in the original non stationary audio signal $x_{n}$ to obtain the watermarked audio $y_{n}$.

Since the watermark $t_{n}$ is piecewise stationary, how does it modify the stationarity (or the non stationarity) of the original audio signal? And precisely, is the watermarked signal, as expected, "more" stationary than the original?

In this paper, we address non stationarities which consist in abrupt changes over short durations in the spectral characteristics of audio signals. Stationarity indices (SI's) based on probabilistic interpretation of time frequency representations (TFR's) [7], [8] computed in case of original and watermarked audio are compared.

The paper is organized as follows: we introduce in section II the considered watermarking scheme and the stationarity indices based on Kolmogorov, Küllback and Bhattacharyya distances. In sections III and IV, we present simulation results obtained with artificial and audio signals, and we introduce an example of application in an acoustic echo canceling system. In section $\mathrm{V}$, we make first conclusions on the watermarking influence on the stationarity of audio signals. 


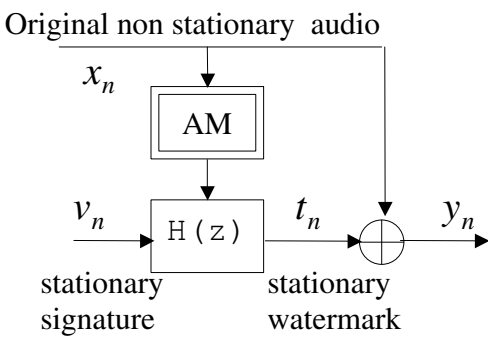

Fig. 1. The considered time domain perceptual watermarking scheme.

\section{WATERMARKING AND STATIONARITY}

\section{A. The time domain perceptual watermarking scheme}

We consider the time domain perceptual watermarking scheme [9] of Fig. 1. To ensure inaudibility, the embedded watermark signal $t_{n}$ is obtained by spectral shaping of the stationary and white random sequence $v_{n}$ through an allpole filter $H(z)$ of order $P$. The squared frequency response module of $H(z)$ matches with the frequency masking threshold $M_{x}(f)$ of the signal $x_{n}$, over processing windows of $N$ samples.

Two kinds of signals are considered: test signals and audio signals (speech and music). In case of watermarking music signals, the masking threshold $M_{x}(f)$ is computed by an auditory model (AM) and is updated each $N$-samples window. Besides, speech signals can be considered as autoregressive to order $P$ and stationary over durations of ca $20 \mathrm{~ms}$. They can be modeled as the output of an allpole filter with transfer function $1 / A(z)$ excited with white noise. As in perceptual speech coders, we use a perceptual allpole filter $H(z)$, given by $1 / A\left(\frac{z}{\gamma}\right)$, where $\left.\gamma \in\right] 0,1[$ is a weighting factor, to shape the white sequence $v_{n}$ spectrally in order to ensure inaudibility [10]. It is important to note that in both cases (music and speech), the coefficients of the filter $H(z)$ are updated each processing window. Hence, the watermark signal $t_{n}$ can be considered as piecewise stationary over each window.

\section{B. Stationarity indices}

Time-frequency based distance and divergence measures were proposed [11], [12] to detect abrupt spectral changes in non stationary signals, which seem to overcome the limitations of parametric modeling based methods in case of short duration transients. The formal analogy between time frequency distributions (TFD's) and bidimensional probability density functions has been addressed in several works (see for example [13], [14]). This analogy is based on the fact that a large class of TFD's present the following properties:

$$
\begin{aligned}
& \text { energy preservation: } \quad \iint C_{s}(t, f) d t d f=\int|s(t)|^{2} d t \\
& \text { marginal properties: }\left\{\begin{array}{l}
\int C_{s}(t, f) d f=|s(t)|^{2} \\
\int C_{s}(t, f) d t=|S(f)|^{2}
\end{array}\right.
\end{aligned}
$$

where $S(f)$ and $C_{s}(t, f)$ are respectively the Fourier transform and the TFD of the time signal $s(t)$.

The probabilistic similarity given by (1) and (2) suggests the use of Shannon entropy to measure the information content of signals in the time frequency plane [15]. Hence, applied to a

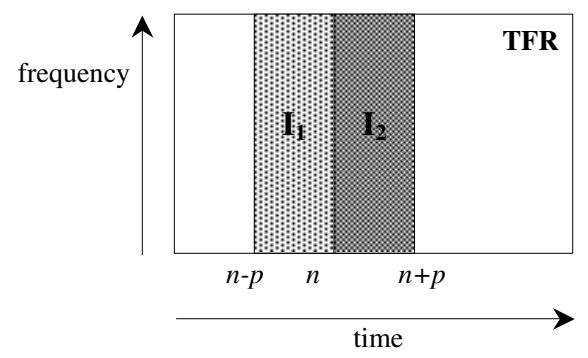

Fig. 2. Subimages $I_{1}$ and $I_{2}$ of the global TFR.

TFR $I(t, f)$ of a unit energy signal, the Shannon entropy is given by:

$$
H(I)=-\iint I(t, f) \log _{2} I(t, f) d t d f
$$

Note that the entropy (3) is defined only for positive valued TFR's. In [16], the authors propose the use of Rényi entropy to circumvent this restriction.

The parallel between TFR and probability theory has been exploited in many fields such as signal segmentation and classification [17].

The method we choose to detect non stationarities in audio signals, proposed in [7], is based on stationarity indices, which consist in distance measures between the same global TFR of the signal but at different instants. The global TFR is computed over the audio signal duration. At each analysis instant $n$, two subimages $I_{1}(n ; \tau, f)$ and $I_{2}(n ; \tau, f)$ with equal duration $p$ are extracted from the global TFR on both sides of the instant $n$, as illustrated on Fig. 2, where:

$$
\begin{aligned}
& I_{1}(n ; \tau, f)=T F R(n-p+\tau, f) \\
& I_{2}(n ; \tau, f)=T F R(n+\tau, f)
\end{aligned}
$$

The parameter $p$ is the duration of each subimage and $\tau \in$ $[0, p]$. Both subimages are normalized to have unit energy as follows:

$$
N I_{k}(n ; \tau, f)=\frac{\left|I_{k}(n ; \tau, f)\right|}{\int_{\tau=0}^{p} \int_{-\infty}^{+\infty}\left|I_{k}(n ; \tau, f)\right| d f d \tau} \quad k=1,2
$$

The normalized subimages are then compared by computing a distance measure, issued from probabilistic time-frequency interpretation. The choice of the couple (distance measure, TFR) is quite difficult considering all possible combinations [12]. Since in the considered application, the frequency accuracy of the energy localization is not of much importance, whereas a high sensitivity of the distance measure over time when an abrupt change in the spectral characteristics occurs is desirable, we choose the smoothed spectrogram for the TFR, which is of simple use, as discussed in [7], with a Hamming smoothing window of length $N_{h}$. Concerning the choice of the distance measure, we investigated the following three distances, all borrowed from probability theory:

\section{The Kolmogorov distance:}

$$
S I_{k o}(n)=\int_{\tau=0}^{p} \int_{-\infty}^{+\infty}\left|N I_{1}(n ; \tau, f)-N I_{2}(n ; \tau, f)\right| d f d \tau
$$


The Küllback divergence:

$$
\begin{array}{r}
S I_{k u}(n)=\int_{\tau=0}^{p} \int_{-\infty}^{+\infty}\left(N I_{1}(n ; \tau, f)-N I_{2}(n ; \tau, f)\right) \\
. \log \left(\frac{N I_{1}(n ; \tau, f)}{N I_{2}(n ; \tau, f)}\right) d f d \tau
\end{array}
$$

The Bhattacharyya distance:

$$
S I_{b h}(n)=-\log \left(\int_{\tau=0}^{p} \int_{-\infty}^{+\infty} \sqrt{N I_{1}(n ; \tau, f) \cdot N I_{2}(n ; \tau, f)}\right) d f d \tau
$$

In probability theory, the Kolmogorov distance and the Küllback divergence are used for the comparison of probability densities. The Bhattacharyya distance, first proposed in a statistical context, was introduced in [18] as a powerful tool in signal selection.

In [12], the authors show experimentally that the Kolmogorov distance is insensitive to noise in detecting abrupt changes in spectral characteristics of test signals. This result was confirmed by the same authors in [8] in case of music signals. Indeed, in our application, where the watermark signal can be considered as a noise, the Kolmogorov distance led to small differences between the $S I_{k o}$ values of watermarked and original signals. Besides, the Bhattacharyya distance and the Küllback divergence showed a much higher sensitivity to the modifications caused by the watermark addition. Thus, the remainder of this paper is based on the use of the Küllback and Bhattacharyya indices.

The evolution of the above discussed stationarity indices over time is interpreted as follows: if the signal characteristics of the analyzed signal present no changes at instant $n$, the stationarity index is near zero, otherwise it significantly peaks indicating the presence of a non stationarity. The parameter $p$ delimits the considered analysis time at each instant $n$ and it allows the selectivity/sensitivity control of the SI's: higher $p$ values lead to smoother SI's.

\section{WATERMARKING INFLUENCE ON THE STATIONARITY OF ARTIFICIAL SIGNALS}

In this section, we show that adding a stationary watermark $t_{n}$ in the time domain modifies the non stationarity property of the original signal.

Since the watermark $t_{n}$ is stationary over $N$-samples windows, we focus our study on the watermarking influence over windows of samples presenting short duration transients.

In the following, we first present simulation results using test signals, which correspond to artificial transients, and their watermarked version. This is done in order to display the behavior of the SI's in this particular context and to set the right values for the sensitivity parameter $p$.

The test signal $x_{n}$ of Fig. 3a has been generated with a sampling frequency $f_{e}=10 \mathrm{kHz}$ and is 512 samples long. The corresponding watermark signal $t_{n}$ has been synthesized, as depicted on Fig. 1, using a perceptual spectral shaping filter of order $P=10$ with a perceptual factor $\gamma=0.8$.

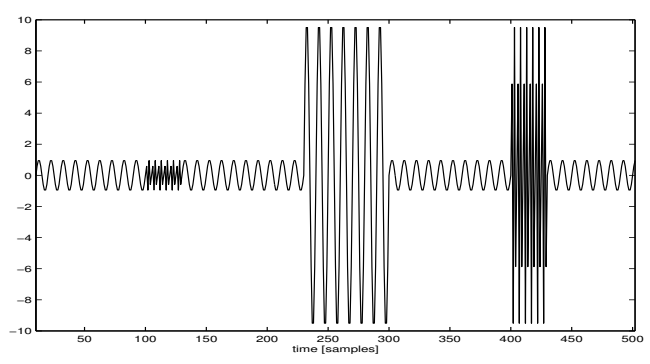

(a) Test signal $x_{n}$

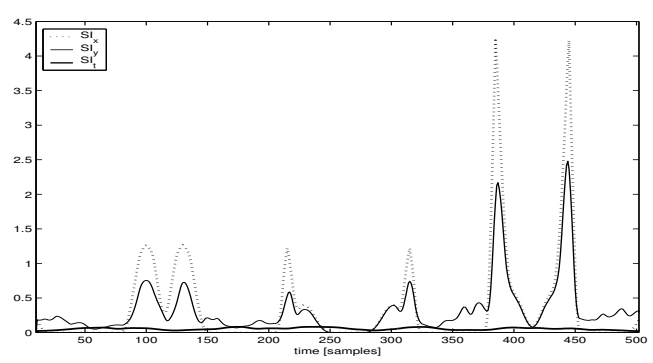

(b) Küllback divergence

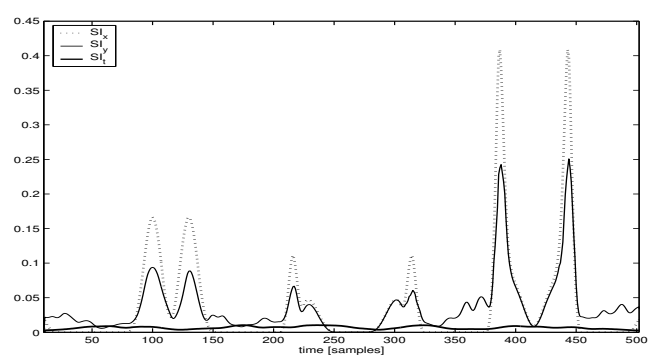

(c) Bhattacharyya distance

Fig. 3. Stationarity indices (b) and (c) of the considered artificial transient $x_{n}$ (dotted line) and of the watermarked signal $y_{n}$ (full line). Simulation settings: $p=10, N_{h}=31, P=10, \gamma=0.8$.

The considered test signal $x_{n}$ presents three transient segments occurring at samples 101, 231 and 431. The first transient segment consists in a short duration frequency jump. The second transient consists in an amplitude variation, and the last transient combines both the first and the second transients: a frequency jump and an amplitude variation. During each segment, the signal $x_{n}$ is composed from only one tone. We define in the following a finite duration tone by $x\left(a, f,\left[n_{1}\right.\right.$ : $\left.n_{2}\right]$ ), where $a$ is the amplitude, $f$ the normalized frequency and $\left[n_{1}: n_{2}\right]$ the time duration. The test signal is then given by:

- stationary zones: $x(1,0.1,[1: 100]), x(1,0.1,[131$ : 230]), $x(1,0.1,[301: 400]), x(1,0.1,[431: 512])$,

- first transient: $x(1,0.4,[101: 130])$,

- second transient: $x(10,0.1,[231: 300])$,

- third transient: $x(10,0.4,[401: 430])$.

We denote respectively $S I_{x}$ and $S I_{y}$ the SI's of the original signal $x_{n}$ and of the watermarked signal $y_{n}=x_{n}+t_{n}$. Fig. 4a shows the added watermark $t_{n}$ and Fig. $4 \mathrm{~b}$ the power spectral densities (PSD's) of the signal $x_{n}$ (full line) and of the corresponding watermark $t_{n}$ (dotted line), with the squared frequency response module of the perceptual filter $H(z)$ (thick line). We compare then the computed Küllback and Bhattacharyya distances of $x_{n}$ and those of its watermarked 


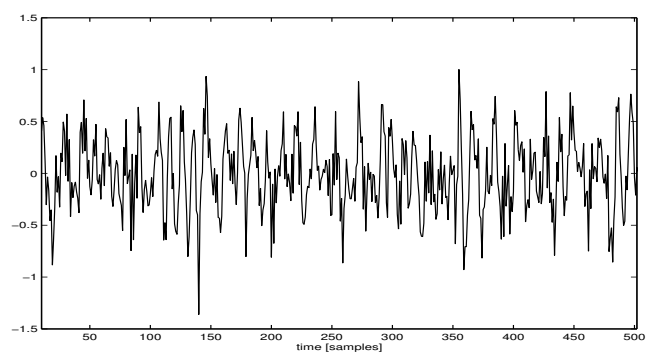

(a)

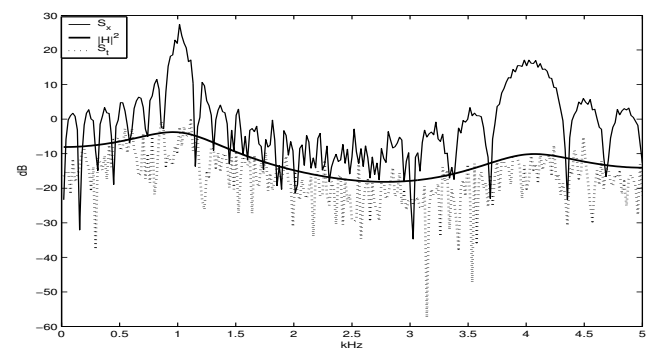

(b)

Fig. 4. (a) Watermark signal $t_{n}$. (b) PSD's of test signal $x_{n}$ (full line), of the watermark $t_{n}$ (dotted line) and squared frequency response module of filter $H(z)$.

version $y_{n}$ on Fig. $3 \mathrm{~b}$ and Fig. $3 \mathrm{c}$ respectively.

As expected, both SI's have the same behavior: they peak at the transient boundaries and they are near zero elsewhere. Besides, we notice that the SI's react with higher peaks, in case of the third transient. Indeed, the non stationarity of the third transient concerns both the frequency content and the power of the signal $x_{n}$.

The sensitivity parameter $p$ should not be greater than $\Delta n / 2$, where $\Delta n$ is the duration between successive transient boundaries, otherwise successive SI peaks will overlap and reduce the detection accuracy of each transient instant.

The thick line curve in Fig. 3b and Fig. 3c corresponds to the SI of the watermark signal $t_{n}$. As expected, it is near zero over the $\mathrm{N}$ signal samples, because $t_{n}$ is stationary.

The main conclusion is that, on both figures, the SI in the watermarked case has been significantly decreased (to about $50 \%$ ) at the transient boundaries.

\section{WATERMARKING INFLUENCE ON THE STATIONARITY OF AUDIO SIGNALS}

Music can be considered as a succession of periods of relative stability, in spite of the presence of transient attacks, such as percussion, inducing high frequency noise [19].

Speech is rather a rapid succession of noise periods, such as unvoiced consonants, periods of relative stability as vowels, and periods of silence.

Note that, since the watermark signal is not as dynamic as the host signal, it may affect the acoustic quality of the content. Indeed, any watermarking techniques invariably perturb the statistics, thus the audio quality of the cover signal. Usually, a trade-off between audio quality or watermark imperceptiveness, robustness, security and complexity, is made

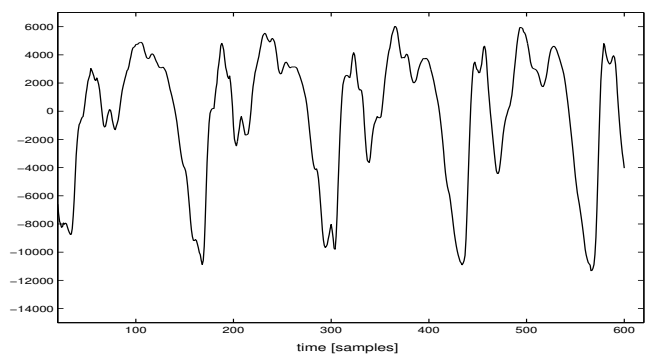

(a) voiced segment (vowel " $a$ ")

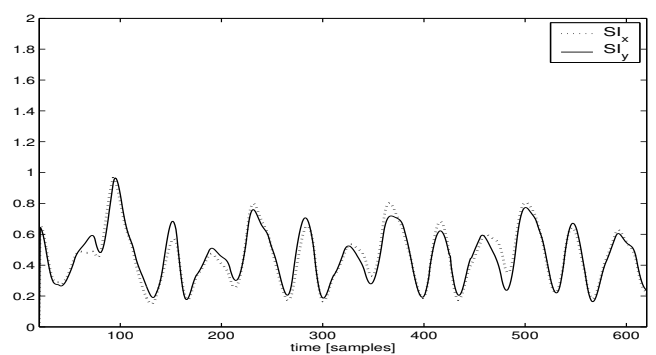

(b) Küllback divergence

Fig. 5. (a) Voiced segment $x_{n}$ and (b) stationarity indices of $x_{n}$ (dotted line) and of the watermarked signal $y_{n}$ (full line): no stationarity improvement of voiced speech segments. Simulation settings: $p=20, N_{h}=61, P=20$, $\gamma=0.8$.

for the aimed application (multimedia copyright protection applications, hidden communications over a public audio channel, audio broadcast monitoring,...). Since most of the watermarking systems rely on the imperfect human auditory system, several objective audio quality measures are perceptual (Bark Spectral Distortion, Perceptual Audio Quality,...); they incorporate frequency masking threshold to differentiate between audible and inaudible distortion [20]. Recently, the ITU standardized an objective quality measure [21]: $\mathrm{PEAQ}^{1}$.

The time domain perceptual watermarking scheme used in this paper is based on the MPEG $^{2}$ psychoacoustic model No1 for the music signals and the perceptual filtering technique for speech signals. The audio quality of the watermarked signals was subjective tested.

In this section, we present a comparative study of the SI's in case of original and watermarked speech and music. The used audio signals are sampled at $f_{e}=32 \mathrm{kHz}$.

In the music case, the AM updates the frequency masking threshold each $N=512$ sample blocks, thus the considered processing window has a duration of $16 \mathrm{~ms}$. In the speech case, the perceptual filter $H(z)$ is updated each $N=640$ samples, corresponding to processing windows of $20 \mathrm{~ms}$.

\section{A. Speech signal}

In case of speech signals, we consider transitions between unvoiced to voiced zones as transients.

We analyze first on Fig. 5a and Fig. 6a respectively $20 \mathrm{~ms}$ of voiced and unvoiced segments separately. We notice neither significant enhancement nor degradation of the stationarity from the SI's of Fig. 5b and Fig. 6b. Indeed, both Küllback

\footnotetext{
${ }^{1}$ PEAQ: Perceptual Evaluation of Audio Quality.

${ }^{2}$ MPEG: Moving Picture Expert Group.
} 


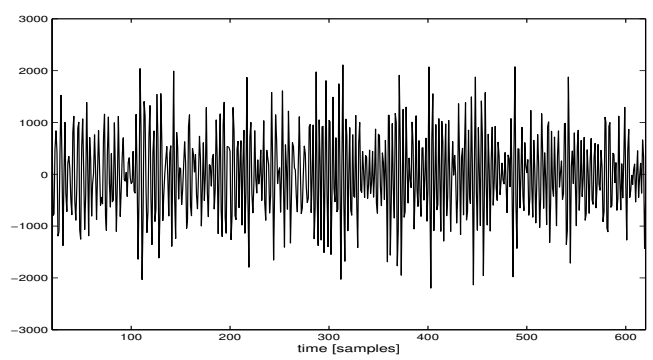

(a) unvoiced segment (consonant " $s$ ”)

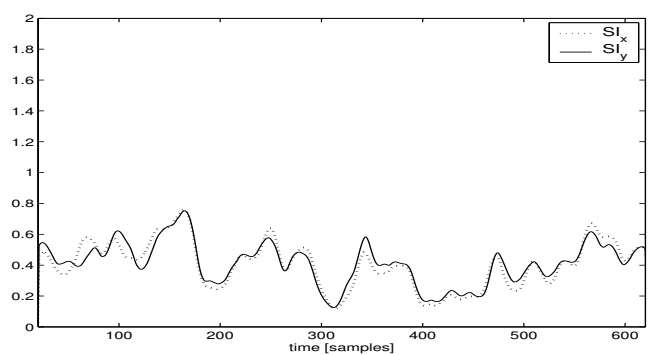

(b) Küllback divergence

Fig. 6. (a) Unvoiced speech segment $x_{n}$ and (b) stationarity indices of $x_{n}$ (dotted line) and of the watermarked signal $y_{n}$ (full line): no stationarity enhancement of unvoiced segments. Simulation settings: $p=20, N_{h}=61$, $P=20, \gamma=0.8$.

based SI's, $S I_{x}$ and $S I_{y}$, are approximately equal and they have small values. That is because noise like unvoiced segments and harmonic voiced segments can be considered as stationary.

On Fig. 7a, we consider a segment of the syllable "sa" to analyze the unvoiced/voiced transition. Fig. 7b shows the corresponding watermark signal $t_{n}$. As expected, the value of the Küllback index $S I_{y}$ has been significantly reduced at the transition area (about $n=220$ ), as shown on Fig. 7c. This result confirms the influence of the watermark on the stationarity of audio signals over transient segments.

In the following section, we analyze music signals. We will see that the stationarity enhancement after watermarking is more important. Indeed, attack transients are more present in music.

\section{B. Music signals}

Music attacks correspond to the beginning of notes produced by an instrument. They are areas of short duration energy (about a few ms) with rapid variations of the sound signal. Particularly, attacks are accompanied by an abrupt short time energy increase distributed on the whole spectrum and noticeable in the high frequencies, since energy is usually concentrated in the low ones [19].

We analyze in the following two examples of transients over durations of $16 \mathrm{~ms}$. The first transient segment is an excerpt from the song Lucille of Michel Jonasz (Fig. 9a). It corresponds to a percussion attack accompanied by the consonant " $p$ ". Indeed, we note on Fig. 8b, the high frequency noise caused by the percussion. The corresponding watermark $t_{n}$ is shown on Fig. 8a.

The second example is an indian zither excerpt and is depicted on Fig. 11a. The corresponding watermark signal is shown on

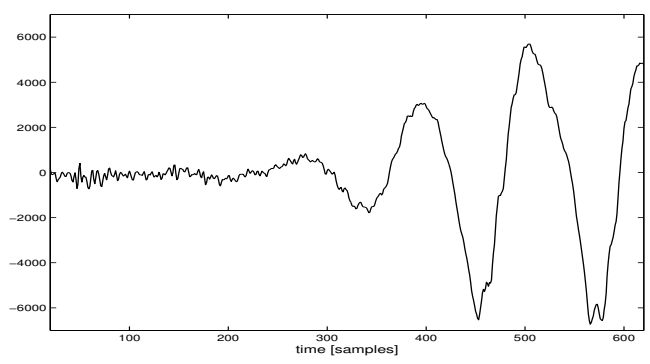

(a) transient segment

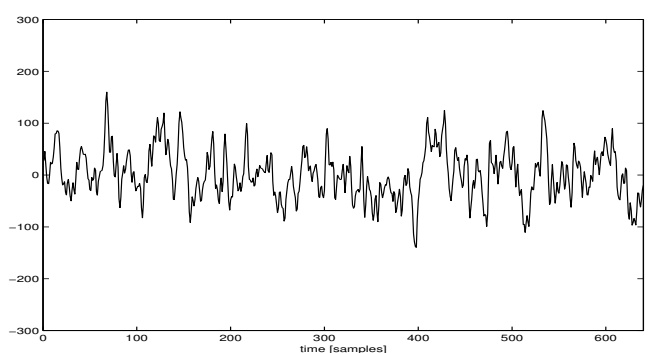

(b) watermark signal $t_{n}$

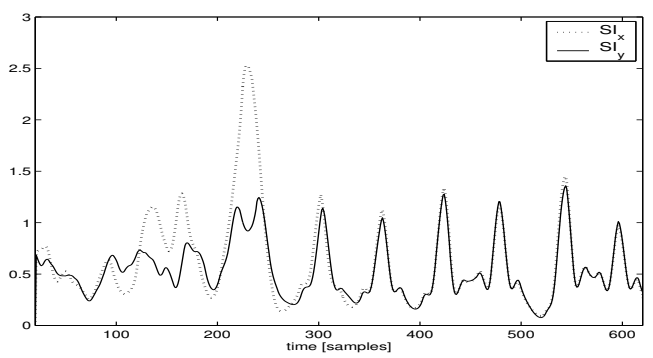

(c) Küllback divergence

Fig. 7. (a) Unvoiced/voiced transition (syllable "sa"). (b) Corresponding watermark signal. (c) $S I_{x}$ (dotted line) and $S I_{y}$ (full line): Stationarity improvement to ca 50\%. Simulation settings: $p=20, N_{h}=61, P=20$, $\gamma=0.8$.

Fig. 10a. The transient of this example is caused by a note attack, resulting too in a high frequency noise as shown on Fig. 10b.

Fig. 9b and Fig. 9c show the important decrease of the $S I_{y}$ value compared to that of $S I_{x}$ at the attack instant $(n=200)$ for both the Küllback and the Bhattacharyya distances.

Similarly, for the zither attack of Fig. 11a, which PSD is depicted on Fig. 10b with the PSD of the watermark $t_{n}$ (dotted line), we note that the stationarity index $S I_{y}$ of the watermarked audio has been significantly reduced to more than $50 \%$ of the stationarity index $S I_{x}$ of the original audio for both distances of Fig. 11b and Fig. 11c.

As in the speech case, no noticeable stationarity enhancement is achieved for harmonic music segments, however, the stationarity indices have been significantly reduced for transient attack segments.

\section{Example of application: robust acoustic echo cancelation}

In audio- and visio-conferencing, the communication quality is altered by the acoustic coupling between loudspeakers and microphones, which results in an echo transmitted through the microphones. The echo is the result of the convolution of the loudspeaker output (the received signal) with the impulse 


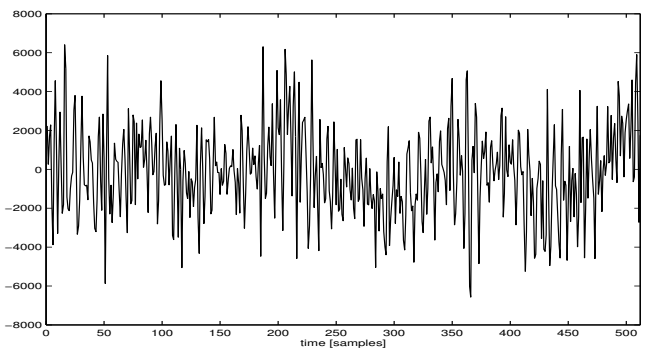

(a)

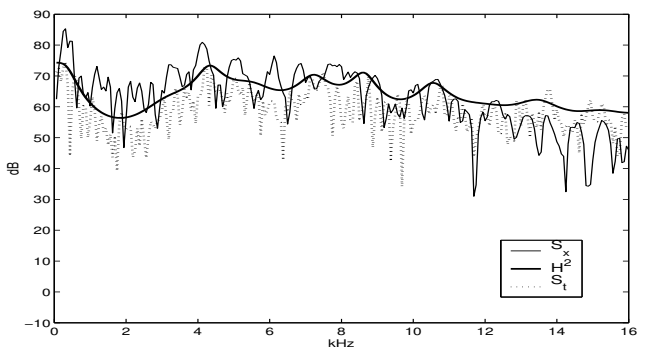

(b)

Fig. 8. (a) watermark signal $t_{n}$. (b) PSD's of audio excerpt $x_{n}$ (song of M. Jonasz: Lucille) and of the watermark $t_{n}$ (dotted line) with frequency response $|H|^{2}$ (thick full line).

response (IR) of the conference room. The role of an adaptive acoustic echo canceler (AEC) is to identify the IR of the echo path in order to reduce the echo.

The principle of the generic time domain monophonic AEC is depicted on Fig. 12 (full line scheme: $t_{n}=0$ ). The taps of the AEC $G_{n}$ are updated according to the residual echo $e_{n}=y_{n}-\hat{y}_{n}$, where $y_{n}$ is the echo and the output $\hat{y}_{n}$ is the estimated echo. The AEC input is the received audio $x_{n}$.

It is well known that adaptive algorithms are very sensitive to the non stationarity of the input. Indeed, the adapting algorithm interprets peaky variations of the residual echo as variations of the channel [22], [23], thus the algorithm sets in tracking mode to pursue those variations. This situation results in the degradation of the AEC performance, which is equivalent to an altered quality of the transmitted signal after echo removal.

The AEC sensitivity concerns both abrupt amplitude and frequency variations. Power normalized adaptive algorithms (for example the $\mathrm{NLMS}^{3}$ ) reduce the amplitude variation impact only in the mean, and not locally at the transient zone [22], [23]. Similarly, the affine projection algorithm (APA) [24] reduces the effect of variations of the frequency content only in the mean and not locally.

The proposed preprocessing by adding a watermark was tested with a monophonic AEC adapted with the NLMS algorithm [6]. The modified AEC is depicted on Fig. 12, where the dotted lines represent the preprocessing part: the addition of the stationary watermark $t_{n}$ to the received audio $x_{n}$. The AEC input is then the watermarked signal $z_{n}=x_{n}+t_{n}$. The AEC of Fig. 12 was tested with speech signals, hence the transfer function $H(z)$ is a perceptual filter as described in section II-A. The input to $H(z)$ is a white random and

${ }^{3}$ NLMS: Normalized Least Mean Squares

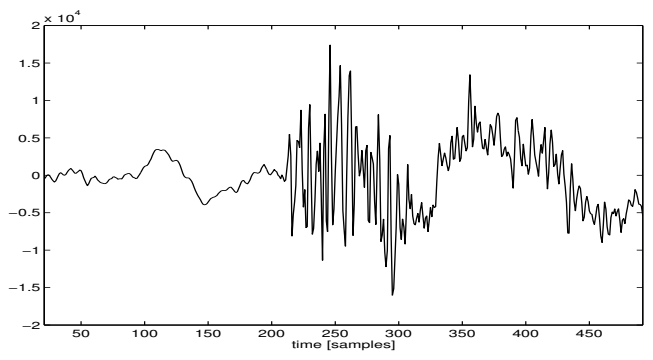

(a) Transient segment $x_{n}$

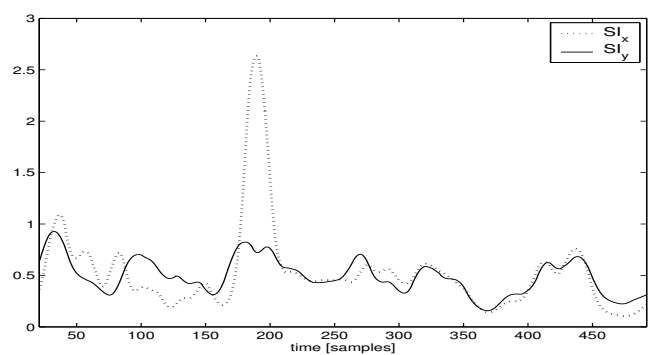

(b) Küllback divergence

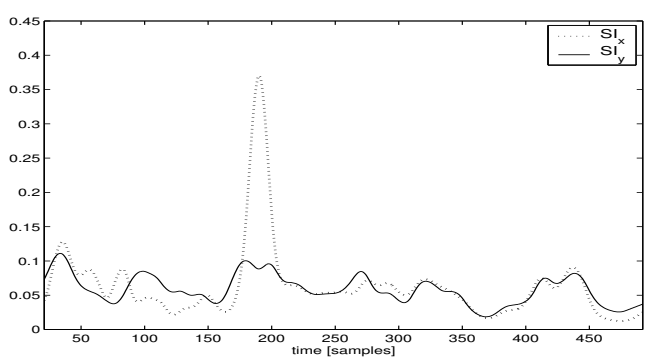

(c) Bhattacharyya distance

Fig. 9. (a) Percussion attack segment $x_{n}$ and corresponding SI's (b) and (c): significant stationarity improvement. Simulation settings: $p=20, N_{h}=61, P=20$.

stationary sequence $v_{n}$. The performance of an AEC is usually measured by the instantaneous $\mathrm{ERLE}^{4}$, given by:

$$
\mathrm{ERLE}=\frac{P_{n}^{y}}{P_{n}^{e}}
$$

where $P_{n}^{y}$ and $P_{n}^{e}$ are the power values of $y_{n}$ and $e_{n}$ respectively, computed over sliding sample windows. Preliminary and convincing results were obtained [6]. Indeed, the enhancement of the ERLE using the proposed preprocessing step reached ca $5 \mathrm{~dB}$ in the steady state mode. The preprocessing improved the robustness of the AEC to short segment transients and led to the stabilization of the ERLE, insuring a nearly constant audio quality.

\section{CONCLUSION}

In this paper, audio watermarking is analyzed from an unusual point of view, where the information content of the watermark is not relevant. We present a study of the time domain perceptual watermarking influence on the stationarity of audio signals. Indeed, since the embedded watermark is piecewise stationary (i.e. over processing windows of $\mathrm{N}$ samples), it modifies the stationarity of the original audio signal. 


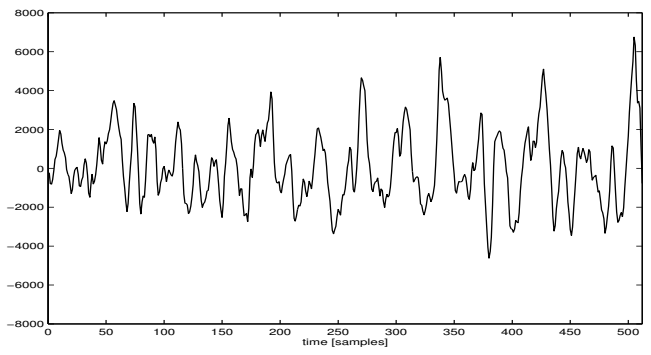

(a)

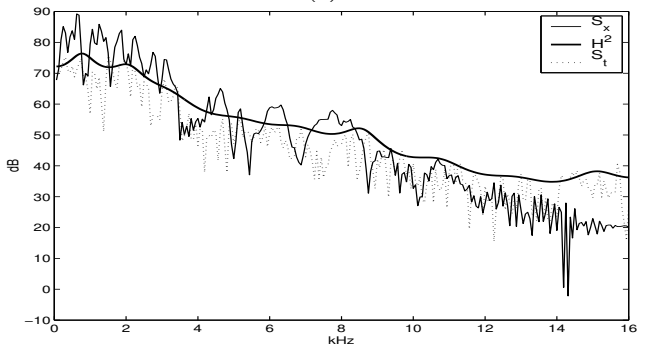

(b)

Fig. 10. (a) Watermark signal $t_{n}$. (b) PSD's of indian zither attack excerpt $x_{n}$ and of corresponding watermark $t_{n}$ (dotted line). The thick line curve represents the frequency response $|H|^{2}$.

The used non stationarity measure is based on probabilistic interpretation of TFR's and stationarity indices. Simulation results with two kinds of signals, test and audio signals, show a significant stationarity enhancement of short segments presenting transient attacks. This enhancement is limited to transient areas and is more important in case of music, since attacks are more present in those signals. Thus, audio watermarking can be viewed as a way to modify audio signal statistics, in order to enhance the performances of audio processing systems.

\section{ACKNOWLEDGMENT}

The authors would like to present their thanks to Professor C. Doncarli and M. Davy (IRCCyN, Nantes, France) for their helpful advises.

The authors are grateful to the reviewers for their constructive comments.

\section{REFERENCES}

[1] D. Kirovski and H. Malvar, "Spread-spectrum watermarking of audio signals," IEEE Trans. on Signal Processing, vol. 51, no. 4, pp. 10201033, 2003.

[2] J. Doherty and R. Porayath, "A robust echo canceler for acoustic environments," IEEE Trans. on Circuits and Systems II: Analog and Digital Signal, vol. 44, no. 5, pp. 389-396, 1997.

[3] R. Boyer, S. Essid, and N. Moreau, "Non stationary signal parametric modeling techniques with an application to low bit rate audio coding," in Proc. of the 6th IEEE Int. Conf. on Signal Processing, 2002.

[4] A. Gilloire and V. Turbin, "Using auditory properties to improve the behaviour of stereophonic acoustic echo cancellers," in Proc. of IEEE Int. Conf. on Acoustics, Speech and Signal Processing, 1998.

[5] S. Larbi and M. Jaïdane, "Watermarking influence on the stationarity of audio signals," in Proc. of IEEE Int. Conf. on Acoustics, Speech and Signal Processing, Hong Kong, 2003.

[6] S. Larbi, M. Jaïdane, M. Turki, and M. Bonnet, "On the robustness of an echo canceler robust to speech non stationarities," in CORESA (COmpression et REpresentation des Signaux Audiovisuels), in french, France, 2001.

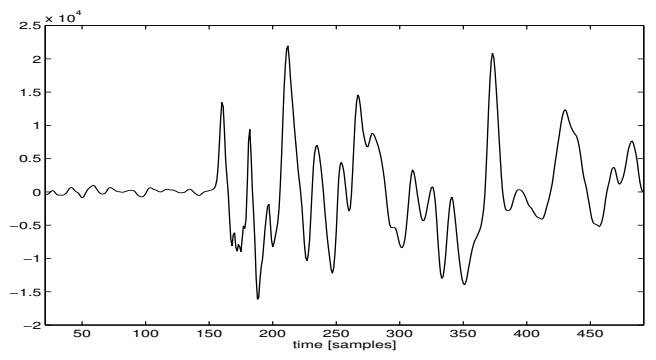

(a) Transient zither attack $x_{n}$

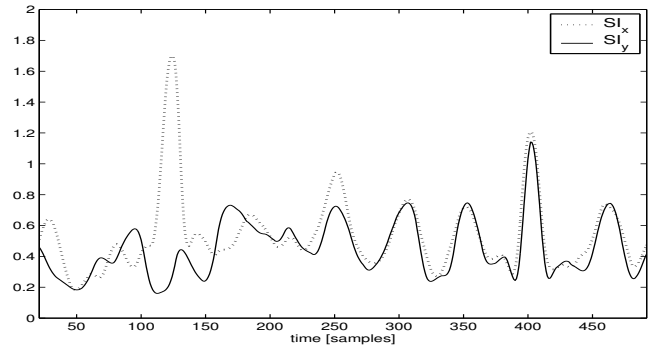

(b) Küllback divergence

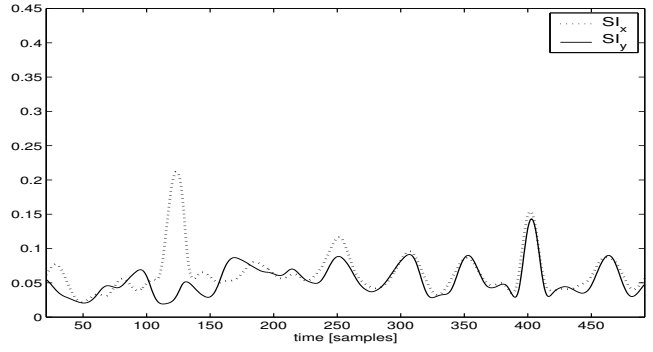

(c) Bhattacharyya distance

Fig. 11. (a) Example of indian zither note attack: the watermark reduced the SI's (b) and (c) to about 50\%. Simulation settings: $p=20, N_{h}=61$, $P=20$.

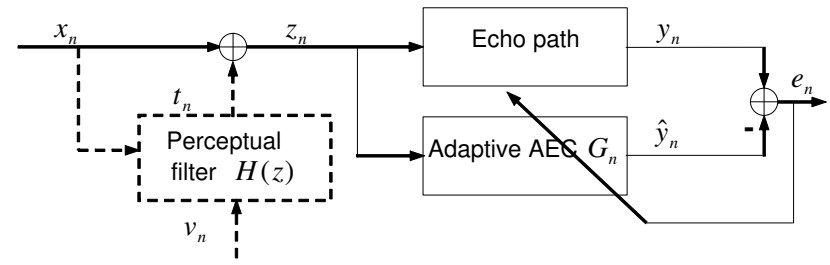

Fig. 12. Time domain AEC system (full line scheme). The dashed part represents the preprocessing step by adding a watermark $t_{n}$ to the received audio $x_{n}$.

[7] H. Laurent and C. Doncarli, "Stationarity index for abrupt changes detection in the time-frequency plane," IEEE Signal Processing Letters, vol. 5, no. 2, pp. 43-45, 1998.

[8] H. Laurent, M.-F. Lucas, and E. Hitti, "Abrupt changes detection in the time-scale and in the time-frequency planes: a comparative study," in Proc. IEEE Int. Symp. on Time-Frequency and Time-Scale Analysis, 1998, pp. 501-504.

[9] L. Boney, A. H. Tewfik, and K. N. Hamdy, "Digital watermarks for audio signals," in IEEE Int. Conf. on Multimedia Computing and Systems, Japan, 1996.

[10] A. M. Kondoz, Digital speech, coding for low bit rate communications systems, Wiley, Ed., 1995.

[11] O. Michel, R. G. Baraniuk, and P. Flandrin, "Time-frequency based distance and divergence measures," in Proc. IEEE Int. Symp. on Signal processing, 1994, pp. 64-67.

[12] H. Laurent and C. Doncarli, "Abrupt changes detection in the timefrequency plane," in Proc. IEEE Int. Symp. on Time-Frequency and TimeScale Analysis, 1996, pp. 285-288.

[13] L. Cohen, "Time-frequency distributions - A review," Proc. IEEE, 
vol. 77, no. 7, 1989.

[14] W. Williams, M. Brown, and A. Hero, "Uncertainty, information, and time-frequency distributions," Proc. SPIE, Advanced Signal Processing, Algorithms, Architecture and Implementations II, vol. 1566, 1991.

[15] P. Flandrin, R. G. Baraniuk, and O. Michel, "Time-frequency complexity and information," in Proc. of IEEE Int. Conf. on Acoustics, Speech and Signal Processing, 1994.

[16] R. G. Baraniuk, P. Flandrin, A. Janssen, and O. Michel, "Measuring time-frequency information and complexity content using the Rényi entropies," IEEE Trans. on Information Theory, vol. 47, no. 4, pp. 13911409, 2001.

[17] I. Vincent, C. Doncarli, and E. Le Carpentier, "Non stationary signals classification using time frequency distributions," in in Proc. IEEE Int. Symp. on Time Frequency and Time Scale Analysis, 1994.

[18] T. Kailath, "The divergence and Bhattacharyya distance measures in signal selection," IEEE Trans. on Communication Technology, vol. COM-15, no. 1, pp. 52-60, 1967.

[19] X. Rodet and F. Jaillet, "Detection and modeling of fast attack transients," in Int. Computer Music Conf., Cuba, 2001.

[20] C. Neubauer and J. Herre, "Digital watermarking and its influence on audio quality," 105th Convention of the Audio Engineering Society, San Francisco, 1998.

[21] M. Van der Veen, F. Brueckers, J. Haitsima, T. Kalker, A. N. Lemma, and W. Oomen, "Multifunctional and high quality audio watermarking technology," 110th Convention of the Audio Engineering Society, Amsterdam, 2001.

[22] S. Haykin, Adaptive Filter Theory. Prentice-Hall, 1991.

[23] O. Macchi, Adaptive processing: The least mean square approach with applications in transmission. Wiley, 2000.

[24] M. Tanaka and Y. Kaneda, "An interpretation of the Affine Projection Algorithm (APA) as the Normalized Least Mean Squares (NLMS) algorithm complex," in Proc. of the IEEE Workshop on Acoustic Echo and Noise Control, USA, 1999.
Sonia Larbi recieved the M. Sc. degree in electrical engineering from the Universität Erlangen Nürnberg (Germany) in 1996. Since 2001, she has been with Ecole Nationale d'Ingénieurs de Tunis (ENIT) in Tunisia, where she is currently assistant teaching at Communications and Information Technologies Department. She is PhD student at ENIT and Ecole Nationale Supérieure des Télécommunications of Paris (France). She is member of the "Unité Signaux et Systèmes" (ENIT). Her research interests are in audio processing and especially in audio watermarking.

Mériem Jaïdane received the M.Sc. degree in electrical engineering from Ecole Nationale d'Ingénieurs de Tunis (ENIT), Tunisia in 1980. From 1980 to 1987, she has worked as research engineer at the "Laboratoire des Signaux et Systèmes", CNRS/Ecole Supérieure d'Electricité, France. She received the Doctorat d'Etat degree in 1987. Since 1987, she has been with ENIT where she is currently a full Professor at Communications and Information Technologies Department (ENIT). She is member of the "Unité Signaux et Systèmes" (ENIT). Her teaching and research interests are in adaptive systems for digital communications and audio processing. 